\title{
Note on the Complete Jacobian Elliptic Integrals.
}

\author{
By F. Bowman, M.A., M.Sc.Tech.
}

(Read 13th December 1918. Received 26th December 1918.)

The complete elliptic integrals $K$ and $E$ are functions of their modulus $k$ which satisfy the equations

$$
\begin{aligned}
k k^{\prime 2} \frac{d K}{d k} & =E-k^{2} K \\
k \frac{d E}{d k} & =E-K
\end{aligned}
$$

respectively.

If we write $\Pi$ for $\Pi(K, a ; k)$ where

$$
\Pi(u, a ; k) \equiv \int_{0}^{u} \frac{k^{2} s n a c n a d n a s n^{2} u}{1-k^{2} s n^{2} a s n^{2} u} d u
$$

is the elliptic integral of the third kind, then $\Pi$ is a function of $a$ and $k$, and $\frac{d \Pi}{d k}$ has a meaning only when $a$ and $k$ are connected by a functional relation. In this note the value of the derivate $\frac{d \Pi}{d k}$ is found in certain cases, on the assumption that such a relation does exist. On account of their simplicity, the results appear to be worth recording. They arose in discussing the geodesics on an ellipsoid of revolution.

Write $x=a \sin \theta \cos \phi$

$$
\begin{aligned}
& y=a \sin \theta \sin \phi \\
& z=c \cos \theta
\end{aligned}
$$

to define a point on the ellipsoid $\frac{x^{2}+y^{2}}{a^{2}}+\frac{z^{2}}{c^{2}}=1$, and suppose $c>a$ and $c^{2} e^{2}=c^{2}-a^{2}$ (prolate spheroid). 
Consider the geodesic which touches the parallels of latitude $\theta= \pm \alpha$. The first integral of geodesics on a surface of revolution is known to be $r \sin \eta=$ const, where $\eta$ is the angle between the curve and the meridian. Expressing this fact for the above surface, we obtain for the equation of the geodesic in question

$$
d \phi=\frac{c}{a} \frac{\sin \alpha}{\sin \theta} \cdot \frac{\sqrt{1-e^{2} \cos ^{2} \theta}}{\sqrt{\cos ^{2} \alpha-\cos ^{2} \theta}} d \theta
$$

and the difference in longitude between a turning point of the geodesic and the point where it crosses the equator is

$$
\frac{c}{a} \int_{a}^{\frac{\pi}{2}} \frac{\sin \alpha}{\sin \theta} \frac{\sqrt{1-e^{2} \cos ^{2} \theta}}{\sqrt{\cos ^{2} \alpha-\cos ^{2} \theta}} d \theta=\frac{c}{a} \int_{a}^{\frac{\pi}{2}} f(\theta, \alpha) d \theta=\frac{c}{a} . I \text {, say. }
$$

Now let us find $\frac{d I}{d \alpha}$. We have

$$
\frac{\partial f}{\partial \alpha}=\cos \alpha \sin \theta \frac{\sqrt{1-e^{2} \cos \theta}}{\left(\cos ^{2} \alpha-\cos ^{2} \theta\right)^{3 / 2}}
$$

and notice that it contains an infinity of order $\frac{3}{2}$ at the lower limit of integration. We can write, however, in this case ( $c f$. Hardy : Quarterly Journal, Vol. 32, 1901)

$$
\frac{d I}{d \alpha}=\lim _{\beta \rightarrow \alpha}\left[\int_{\beta}^{\frac{\pi}{2}} \frac{\partial f}{\partial \alpha} d \theta-f(\beta, \alpha)\right]
$$

Now transform to the notation of the Jacobian elliptic functions by writing

$$
\cos \theta=\cos \alpha \cdot \operatorname{sn}(u+K)=\cos \alpha \frac{c n u}{d n u}, \text { where } k=e \cos \alpha ;
$$

we get

$$
\begin{gathered}
f(\theta, \alpha)=\frac{1}{\cos \alpha} \cdot \frac{d n u}{s n u} \cdot \frac{1}{\sqrt{1+\cot ^{2} \alpha\left(1-e^{2}\right) \operatorname{sn}^{2} u}} \\
\int \frac{\partial f}{\partial \alpha} d \theta=\frac{1}{\cos \alpha} \int \frac{d u}{s n^{2} u}=\frac{1}{\cos \alpha}\left[u-\frac{c n u d n u}{8 n u}-E(u)\right]
\end{gathered}
$$

and

$$
I=\frac{1-e^{2} \cos ^{2} \alpha}{\sin \alpha} \int_{0}^{K} \frac{d u}{1+\cot ^{2} \alpha\left(1-e^{2}\right) \sin ^{2} u}
$$

and without difficulty $\frac{d I}{d \alpha}=\frac{1}{\cos \alpha} \cdot(K-E)$. 
Substituting for $a$ in terms of $k$ throughout this equation we arrive at the result

$$
\frac{d}{d k} \frac{e k^{\prime 2}}{\sqrt{e^{2}-k^{2}}} \int_{0}^{K} \frac{d u}{1+k^{2} \frac{1-e^{2}}{e^{2}-k^{2}} s n^{2} u}=\frac{e(E-K)}{k \sqrt{e^{2}-k^{2}}}
$$

in which we can remove the restriction that $e$ should be less than the unity.

But we can write

$$
\begin{gathered}
I=\frac{e k^{\prime 2}}{\sqrt{e^{2}-k^{2}}} \int_{0}^{K}\left[d u-\frac{k^{2} \frac{1-e^{2}}{e^{2}-k^{2}} s n^{2} u}{1+k^{2} \frac{1-e^{2}}{e^{2}-k^{2}} s n^{2} u} d u\right] \\
=\frac{e k^{\prime 2}}{\sqrt{e^{2}-k^{2}}} \cdot K+i \sqrt{1-e^{2}} \int_{0}^{K} \frac{\frac{i k^{\prime 2} e \sqrt{1-e^{2}}}{\left(e^{2}-k^{2}\right)^{3 / 2}}}{1+k^{2} \frac{1-e^{2}}{e^{2}-k^{2}} s n^{2} u} d u \\
=\frac{e k^{\prime 2}}{\sqrt{e^{2}-k^{2}}} \cdot K+i \sqrt{1-e^{2}} \Pi(K, a ; k)
\end{gathered}
$$

where $8 n^{2} a=-\frac{1-e^{2}}{e^{2}-k^{2}}$.

Taking $I$ in this form and differentiating again with respect to $k$ and equating the result to the right side of (1), we finally obtain

$$
\frac{d \mathrm{II}}{d k}=\operatorname{sn} a \operatorname{cn} a \operatorname{dn} a \cdot \frac{k}{k^{\prime 2}}, k \text {. }
$$

This is the value of $\frac{d \Pi}{d k}$ under the condition $s n^{2} a=-\frac{1-e^{2}}{e^{2}-k^{2}}$, which is easily seen to be equivalent to

$$
\frac{c n^{2} a}{d n^{2} a}=s n^{2}(a+K)=\frac{1}{e^{2}}=\text { const. }
$$

or $\operatorname{sn}(a+K, k)=$ const.

Now if we carry out the same work for the case of the oblate spheroid, using the transformation given by Forsyth (Differential 
Geometry, p 139), we arrive at the result

$\frac{d}{d k} \frac{e}{\sqrt{e^{2}-k^{2}}} \int_{0}^{K} \frac{d n^{2} u}{1+k^{2} \cdot \frac{1-e^{2}}{e^{2}-k^{2}} 8 n^{2} u} d u=\frac{e}{\sqrt{e^{2}-k^{2}}} \frac{E-k^{\prime 2} \cdot K}{k k^{\prime 2}}$

corresponding to (1) above.

After dividing by $e$, subtract (1) from (3) and get

$$
\frac{d}{d k} \frac{1}{\sqrt{e^{2}-k^{2}}} \int_{0}^{I} \frac{d n^{2} u-k^{2}}{1+k^{2} \frac{1-e^{2}}{e^{2}-k^{2}} s n^{2} u} \cdot d u=\frac{1}{\sqrt{e^{2}-k^{2}}} \cdot \frac{k}{k^{\prime 2}}, E
$$

which easily reduces to

$$
\frac{d}{d k} \int_{0}^{K} \frac{\sqrt{e^{2}-k^{2}} \cdot \frac{k^{2}}{e^{2}} \frac{c n^{2} u}{d n^{2} u}}{1-\frac{k^{2}}{e^{2}} \frac{c n^{2} u}{d n^{2} u}} d u=\frac{1}{\sqrt{e^{2}-k^{2}}} \cdot \frac{k}{k^{\prime 2}} E
$$

or, since $\operatorname{sn}(u+K)=\frac{c n u}{d n u}$

and $\operatorname{sn}(2 K-u)=8 n u$

$$
\frac{d}{d k} \int_{0}^{K} \frac{k^{2} \frac{\sqrt{e^{2}-k^{2}}}{e} \cdot \frac{1}{e} \cdot 8 n^{2} u}{1-k^{2} \cdot \frac{1}{e^{2}} \cdot 8 n^{2} u} \quad d u=\frac{1}{\sqrt{e^{2}-k^{2}}} \cdot \frac{k}{k^{2}} . E
$$

Put $\sin a=\frac{1}{e}$, and after multiplying by cn $a$ we get

$$
\frac{d \Pi}{d k}=\frac{\operatorname{sn} a c n a}{d n a} \cdot \frac{k}{k^{\prime 2}} \cdot E \text {. }
$$

This then is the value of $\frac{d \Pi}{d k}$ under the condition sn $a=$ const.

We may notice in passing that the values of $\frac{d K}{d k}$ and $\frac{d E}{d k}$ may be derived as special cases of the equations (1), (3) or (4). For example, putting $e=1$ in (1) we get $k k^{\prime 2} \frac{d K}{d k}=E-k^{\prime 2} K$, and $e=\infty$ gives $k \frac{d E}{d k}=E-K$. 
The results (2) and (5) could have been obtained in a more straightforward manner as follows, though one could hardly have predicted that they would turn out to be as simple as they are.

The expression of $\Pi(u, a ; k)$ in terms of the Jacobian $\theta$ function is $\Pi(u, a ; k)=\frac{1}{2} \log \frac{\theta(u-a)}{\Theta(u+a)}+u . Z(a)$ and on writing $u=K$ it becomes

$$
\Pi(K, a ; k)=K . Z(a)=K . E(a)-a . E .
$$

And since

$$
E(a)=\int_{0}^{a} d n^{2} u d u
$$

and therefore $\frac{d E(a)}{d k}=d n^{2} a \cdot \frac{d a}{d k}+\int_{0}^{a} \frac{\partial}{\partial k}\left(d n^{2} u\right) d u$,

we obtain on differentiating

$\frac{d \Pi}{d k}=E(a) \cdot \frac{d K}{d k}-a \frac{d E}{d k}+\left(K d n^{2} a-E\right) \frac{d a}{d k}+K \int_{0}^{a} \frac{\partial}{\partial k}\left(d n^{2} u\right) d u$.

Now, from the equation $u=\int_{0}^{s n u} \frac{d t}{\sqrt{\left(1-t^{2}\right)\left(1-k^{2} t^{2}\right)}}$

it is easy to prove that, while $u$ remains constant

$$
\frac{\partial s n u}{\partial k}=-\frac{c n u d n u}{k k^{\prime 2}}\left[E(u)-k^{2} \cdot u-k^{2} \frac{8 n u \cdot c n u}{d n u}\right]
$$

and hence that

$$
\frac{\partial d n^{2} u}{\partial k}=\frac{2 k}{k^{\prime 2}}\left[8 n u c n u d n u\left\{E(u)-k^{\prime \prime} \cdot u\right\}+8 n^{2} u d n^{2} u\right]
$$

and after a little reduction we obtain

$$
\int_{0}^{a} \frac{\partial}{\partial k}\left(d n^{2} u\right) d u=\frac{k}{k^{\prime 2}}\left[\operatorname{sna} \operatorname{cn} a d n a-c n^{2} a \cdot E(a)-k^{\prime 2} \cdot a \cdot 8 n^{2} a\right]
$$

Substituting in (7)

$\frac{d \Pi}{d k}=\left(K d n^{2} a-E\right)\left[\frac{d a}{d k}-\frac{1}{k k^{\prime 2}}\left\{E(a)-k^{\prime 2} \cdot a\right\}\right]+\operatorname{sn} a \operatorname{cn} a d n a \cdot \frac{k}{k^{\prime 2}} \cdot K$

This equation gives the value of $\frac{d \Pi}{d k}$ when $a$ and $k$ are connected by any relation of the form $f(a, k)=$ const., provided we introduce the appropriate value of $\frac{d a}{d k}$. 
For example, if we make the assumptions

$$
\text { sn } a=\text { const., } \quad \operatorname{sn}(a+K)=\text { const., }
$$

and evaluate the right side of (11) in each case, we arrive at the results (5) and (2) respectively.

Again, since we are led to define

$$
\Pi(u, a ; k)=\int_{0}^{u} \frac{k^{2} s n a c n a d n a s n^{2} u}{1-k^{2} s n^{2} a s n^{2} u} d u
$$

from a consideration of the integral $\int \frac{\alpha+\beta \operatorname{sn}^{2} u}{1+\gamma \sin ^{2} u} d u$, it is natural to discuss the value of $\frac{d \Pi}{d k}$ when $\gamma\left[=-k^{2} 8 n^{2} a\right]$ remains constant ; that is, when $d n a$ is constant.

Making use of (9) to differentiate $d n^{2} a=$ const., we get

$$
k k^{\prime 2} \frac{d a}{d k}=E(a)-k^{\prime 2} \cdot a-\frac{s n a d n a}{\operatorname{cn} a}
$$

and substituting in (11), we have

$$
\left(\frac{d \Pi}{d k}\right)_{d n a=\text { const. }}=\frac{\operatorname{sna} d n a}{\operatorname{cn} a} \cdot \frac{d K}{d k} .
$$

Finally, when $d u(a+K, k)=$ const., we obtain

$$
k k^{\prime 2} \frac{d a}{d k}=E(a)-k^{\prime 2} \cdot a+\frac{c n a d n a}{\operatorname{sn} a},
$$

and in this case

$$
\left(\frac{d \Pi}{d k}\right)_{d n(a+K)=\text { oonat. }}=-\frac{\text { cn } a d n a}{\operatorname{sn} a} \frac{1}{k^{\prime 2}} \cdot \frac{d E}{d k} .
$$

Collecting the results (11), (5), (2), (12), and (13), we may summarise the foregoing work in the following table :- 


\begin{tabular}{|c|c|}
\hline Relation between $a$ and $k$. & Value of $\frac{d \Pi}{d k}$ \\
\hline$a=$ const. & $\begin{array}{l}\frac{\left(E-d n^{2} a \cdot K\right)}{k k^{\prime 2}}\left(E(a)-k^{\prime 2} \cdot a\right) \\
\quad+\operatorname{sn} a \operatorname{cn} a \operatorname{dn} a \frac{k}{k^{\prime 2}} \cdot K\end{array}$ \\
\hline$s n(a . k)=$ const. & $\frac{\operatorname{snacn} a}{d n a} \cdot \frac{k}{k^{\prime 2}} \cdot E$ \\
\hline sn $(a+K, k)=$ const. & $\operatorname{sn} a c n a d n a \cdot \frac{k}{k^{\prime 2}}, K$ \\
\hline$d n(a . k)=$ const. & $\frac{\operatorname{sn} a d n a}{c n a} \cdot \frac{d K}{d k}$ \\
\hline$d n(a+K, k)=$ const. & $-\frac{c n a d n a}{s n a} \cdot \frac{1}{k^{\prime 2}} \cdot \frac{d E}{d k}$ \\
\hline
\end{tabular}

